\title{
Determinant Factors of the Squat Jump in Sprinting and Jumping Athletes
}

\author{
by \\ Juan José González-Badillo, Pedro Jiménez-Reyes², Jorge Ramírez-Lechuga ${ }^{1}$
}

\begin{abstract}
The aim of this study was to assess the relationship between strength variables and maximum velocity (Vmax) in the squat jump (SJ) in sprinting and jumping athletes. Thirty-two sprinting and jumping athletes of national level $(25.4 \pm 4.5$ years; $79.4 \pm 6.9 \mathrm{~kg}$ and $180.4 \pm 6.0 \mathrm{~cm})$ participated in the study. Vmax in the SJ showed significant relationships with peak force 1 (PF1) ( $r=0.82, p \leq 0.001)$, peak force 2 (PF2) $(r=0.68, p \leq 0.001)$, PF2 by controlling for PF1 ( $r=0.30$, non-significant), the maximum rate of force development at peak force 1 (RFDmax1) $(r=0.62, p \leq$ 0.001), mean RFD 1 (RFDmean1) ( $r=0.48, p \leq 0.01)$, mean RFD 2 (RFDmean2) ( $r=0.70, p \leq 0.001$ ), force at RFDmax $1(r=0.36, p \leq 0.05)$, force at RFDmax $(r=0.83, p \leq 0.001)$ and force at RFDmax 2 by controlling for PF1 $(r$ $=0.40, p \leq 0.05)$. However, Vmax in the SJ was associated negatively with the ratio PF2/PF1 $(r=-0.54, p \leq 0.01)$, time at peak force 2 (Tp2) $(r=-0.64, p \leq 0.001)$ and maximum rate of force development at peak force 2 (RFDmax 2$)(r=-$ $0.71, p \leq 0.001$ ). These findings indicate that the peak force achieved at the beginning of the movement (PF1) is the main predictor of performance in jumping, although the RFDmax values and the ratio PF2/PF1 are also variables to be taken into account when analyzing the determinant factors of vertical jumping.
\end{abstract}

Key words: strength, performance, testing, power.

\section{Introduction}

The vertical jump has been utilized in training and as a predictor of athletic performance (Bosco et al., 1983; Cronin and Hansen, 2005; Vandewalle et al., 1987). It has been observed that some vertical jump variables are related to performance in sprinting (Dowson et al., 1998; López-Segovia et al., 2011; Nesser et al., 1996; Wisløff et al., 2004) and the loss in jumping ability has been considered a good indicator of neuromuscular fatigue caused by repeated high intensity efforts (Gorostiaga et al., 2010; SánchezMedina and González-Badillo, 2011). The height of a squat jump (SJ) has shown a relationship with $60 \mathrm{~m}$ sprint time (Nagahara et al., 2014), acceleration (Nagahara et al., 2014), the Abalakow test, a standing long jump and peak torque (Bosco et al., 1983).

As known by the physics principle,

vertical jump height is directly proportional to the takeoff velocity. Several authors have used takeoff velocity to evaluate vertical jump performance (Kollias et al., 2001; Luhtanen and Komi, 1978; Mohir et al., 2008). This variable cannot be modified once the subject is off the ground (Reiser et al., 2006) and is closely related to maximum velocity (Vmax) (González-Badillo and Marques, 2010). Therefore, the vertical jump depends directly on Vmax (González-Badillo and Marques, 2010). Thus, Vmax could be considered as the best indicator of performance in vertical jumping, as this variable determines the jump height and cannot be voluntary or involuntarily manipulated by the subject, especially if the jump is performed in a vertical guidance because, as known, Vmax is reached milliseconds before the take-off. For this reason, determinant factors of vertical jump

1 - Department of Sport, University of Pablo de Olavide, Seville, Spain.

2 - Department of Physical Activity and Sports Science, Catholic University of San Antonio, Murcia, Spain. 
"practical" performance (actual height reached after the takeoff) are the same that determine Vmax in executing the jump.

Despite knowing the importance of velocity in jump performance, jump height has traditionally been used as an indicator of vertical jump performance (Dowling and Vamos, 1983; González-Badillo and Marques, 2010; Marques and González-Badillo, 2011; Wirth et al., 2015). It is well known that the measurement of jump height is usually estimated through flight time, but this variable may elicit some error due to particular forms of contact with the ground at the time of landing, as well as other deviations in the execution such as an excessive trunk tilt and displacement relative to the baseline. However, Vmax is not influenced by these execution errors when the jump is performed on a Smith Machine with a guide.

Few studies have analyzed the dynamic and kinematic factors able to explain SJ performance (Mackenzie et al., 2014; Marques and González-Badillo, 2011), and they have also used estimated jump height through flight time as performance standard. There is very scarce literature considering Vmax as a performance indicator of the vertical jump. Vmax would be a good indicator of vertical jump performance as it is directly dependent on the factors that determine the outcome, i.e., the applied force and the rate of force development, and, in addition, it cannot be manipulated by the subject. Therefore, the purpose of this study was to assess the relationship between strength variables and maximum velocity (Vmax) in the squat jump in sprinting and jumping athletes. It was hypothesized that Vmax in the SJ, and therefore the reached height, would depend on force peaks and the rate of force development in the two characteristic force slopes of the SJ.

\section{Methods}

\section{Study design}

The present study used a cross-sectional experimental design to examine the relationship between muscle strength variables and $\mathrm{V}_{\max }$ in the squat jump in a group of sprinting and jumping athletes. All the subjects were well conditioned and familiar with testing exercises, which they had been performing regularly as part of training sessions.

\section{Participants}

Thirty-two young men (mean \pm SD: age $25.4 \pm 4.5$ years, body mass $79.4 \pm 6.9 \mathrm{~kg}$, body height $180.4 \pm 6.0 \mathrm{~cm}$ ) participated voluntarily in this study. They were athletes competing in the $100 \mathrm{~m}$ sprint, long jump or triple jump in the Spain Championships. They were familiar with the jumping exercise. The present investigation was approved by the Research Ethics Committee of the Pablo de Olavide University. The subjects received information about the characteristics and goals of the study, the procedures, free participation, possibility of quitting at any moment, and confidentiality of data. Written informed consent was obtained from the subjects before the beginning of the study.

\section{Procedures}

Participants were familiar with the testing procedures since they had been performing the jumping exercise as part of their normal training routine. After a standardized warm-up, participants performed two maximal SJ trials in a Smith Machine while standing on a portable force plate (Isonet, JLML, Madrid, Spain). The bar of the Smith machine had a linear transducer attached (Isocontrol, JLML, Madrid, Spain) which was synchronized with the force plate. The force plate was connected to a portable computer and recorded data at a sample rate of $1000 \mathrm{~Hz}$. The rotary encoder of the linear transducer registered the position of the bar within an accuracy level of $0.0002 \mathrm{~m}$.

Participants stood on the Smith machine and rested the bar $(17 \mathrm{~kg})$ on their shoulders. For the performance of the SJ test, subjects were asked to stand on the center of the force plate in a semisquatting position. All athletes performed two SJ attempts without any countermovement after keeping the semi-squatting position for 2-3 seconds to avoid taking advantage of elastic energy storage. The SJ trials were performed with a knee angle of 90 degrees, which was determined using a handheld goniometer (Q-TEC Electronic Co. Ltd., Gyeonggi-do, Korea). Athletes immediately jumped vertically and explosively. Hands remained holding on to the bar for the entire movement in order to maintain contact between the bar and the shoulders. Any countermovement was forbidden and carefully checked after each trial using force-time curves. Three minutes of rest were provided between 
each trial to minimize the likelihood of fatigue. Only the best attempt (the highest $V_{\max }$ ) was registered for further analysis.

\section{Variables} SJ:

The following variables were analyzed in the

- Peak Force $(\mathrm{N})$. In the force-time curve two force peaks were observed (Figure 1): the first peak was called peak force 1 (PF1) and the second was called peak force 2 (PF2).

- Time at peak force (ms). The time at peak force 1 (Tp1) was the time from the initiation of force production $(F=0)$ until the PF1. The time at peak force 2 (Tp2) was the time from the initiation of force production until the PF2.

- Maximum RFD $\left(\mathrm{N} \cdot \mathrm{s}^{-1}\right)$. The maximum RFD (RFD max) or maximum slope was the maximum force production per unit of time throughout the force production measured at time intervals of $50 \mathrm{~ms}$. The RFD $\max 1$ corresponded to the slope of the PF1 and the RFDmax2 corresponded to the slope of the PF2 (Figure 1).

- Force at RFDmax $(\mathrm{N})$. It was the applied force in absolute value at the time to reach maximum RFD. The force at RFDmax 1 and RFD max2 was recorded.

- Mean RFD $\left(\mathrm{N} \cdot \mathrm{s}^{-1}\right)$. Two values of mean RFD were calculated. Mean RFD until PF1 (RFDmean1) was the relationship between the PF1 and the time from the initiation of force production until the PF1. Mean RFD until PF2 (RFDmean2) was the relationship between the PF2 and the time from the initiation of force production until the PF2.

- Distance of flexion $(\mathrm{cm})$. It was the distance that the bar went down vertically when the subject maintained a knee flexion of about 90 degrees.

\section{Statistical analysis}

Descriptive statistics are expressed as means \pm SD. The interclass correlation coefficient (ICC) was used to determine between-subject reliability of jumping tests. The ICC was calculated with the one-way random effects model. The standard error of measurement was calculated as the root mean square of total mean square intra-subject. This standard error was used for the calculation of the coefficient of variation (CV). Correlations were determined using Pearson's coefficients (r). To isolate the possible effect of body mass, height and distance of flexion on physical performance, these relationships were also adjusted with partial correlations $\left(\mathrm{r}_{\mathrm{w}}\right)$. Statistical significance was accepted at $p \leq 0.05$ for all analyses. The statistical analysis was performed using SPSS 20.0 (SPSS, Chicago, IL).

\section{Results}

Vertical jump measurement showed good stability (reliability). Test-retest reliability for $\mathrm{V}_{\max }$ as measured by the $\mathrm{CV}$ was $3 \%$ and the ICC (95\%CI) was 0.84 [0.67-0.92].

Mean values, SD and range of the different variables assessed are presented in Table 1. PF1 was always less than PF2, however, RFDmax1 was greater than RFD max2.

The present study found significant relationships between SJ $\mathrm{V}_{\max }$ and several variables of strength. All variables except $\mathrm{Tp} 1$ showed a significant correlation with $\mathrm{V}_{\max }$ (Table 2). $\mathrm{V}_{\max }$ correlated positively with $\mathrm{PF} 1(\mathrm{r}=0.82, p \leq$ $0.001), \mathrm{PF} 2(\mathrm{r}=0.68, p \leq 0.001), \mathrm{RFD}_{\max 1}(\mathrm{r}=0.62, p$ $\leq 0.001)$, RFDmean1 $(\mathrm{r}=0.48, p \leq 0.01)$, RFDmean2 $(\mathrm{r}=$ $0.70, p \leq 0.001)$, force at RFD $\max (\mathrm{r}=0.36, p \leq 0.05)$ and force at RFD $\max _{2}(\mathrm{r}=0.83, p \leq 0.001)$. However, $\mathrm{V}_{\max }$ was associated negatively with the ratio PF2/PF1 ( $\mathrm{r}=-0.54, p \leq 0.01), \mathrm{Tp} 2(\mathrm{r}=-0.64, p \leq$ $0.001)$ and $\operatorname{RFD}_{\max 2}(\mathrm{r}=-0.71, p \leq 0.001)$. The partial correlation between $S J V_{\max }$ and PF2 by controlling for PF1 was 0.30 (non-significant), and the partial correlation between $S J V_{\max }$ and the force at RFD $_{\max 2}$ by controlling for PF1 was $0.40(p \leq 0.05)$. Results did not change substantially by applying partial correlations controlling for body mass, height or distance of flexion.

\section{Discussion}

The aim of the present study was to assess the relationship between strength variables and $\mathrm{V}_{\max }$ in the squat jump in sprinting and jumping athletes. $\mathrm{V}_{\max }$, considered a jumping performance indicator, showed good inter-subject reliability (ICC $=0.84, \mathrm{CI}: 0.67-0.92)$ despite low variability of the sample in this variable ( $\mathrm{CV}=6.69 \%)$, but it showed very good intra-subject reliability with a CV between measures of only $3 \%$.

Results of this study reveal that the best performance predictor to explain jumping ability is PF1. This peak force occurs at few milliseconds after the initiation of jump displacement, that is to say, immediately after completing the static phase 
of movement (Figure 1). The relationship between $\mathrm{V}_{\max }$ and PF1 is positive $(\mathrm{r}=0.82, p \leq 0.001)$, so the higher $\mathrm{PF} 1$, the higher the $\mathrm{V}_{\max }$ tends to be. If we also note that the maximum RFD, which occurs in the slope of the first peak force (RFDmaxi), showed a significantly positive correlation with $\mathrm{V}_{\max }(\mathrm{r}=$ $0.62, p \leq 0.001)$, and that this value is reached before the initiation of movement (Figure 1) in the static phase of movement (jump), our results suggest that the reached height in the ascending phase of flight time (performance from a practical standpoint) depends mostly and directly on what happened before the initiation of displacement in jumping and in the first milliseconds of displacement.

Other studies have concluded that peak power is the best predictor of vertical jump performance, both the SJ (Ashley and Weiss, 1994) and CMJ (Dowling and Vamos, 1993). In the present study, we did not use this variable (peak power) as a possible predictor, since reaching a greater height with the same load would involve a faster displacement of this load, and therefore, it necessarily would have achieved higher power, thus, the relationship between power and jump height would always be positive and high. Therefore, we understand that peak power should not be considered as a predictor, but as a performance variable. Furthermore, we must consider that the reached power in a jump is a consequence of the rest of dynamic variables that have been previously expressed, and therefore, it will never be the cause of a jump, but, in any case, a dependent indicator variable of the jump performance if the weight was always constant.

Table 1

Values of selected performance variables in the squat jump with $17 \mathrm{~kg}(n=32)$. Data presented as mean $\pm S D$ (range).

\begin{tabular}{|c|c|c|c|}
\hline $\begin{array}{l}V_{\max } \\
\left(\mathrm{m} \cdot \mathrm{s}^{-1}\right)\end{array}$ & $2.99 \pm 0.2(2.61-3.27)$ & $\mathrm{RFD}_{\max 1}\left(\mathrm{~N} \cdot \mathrm{s}^{-1}\right)$ & $8068.6 \pm 2303.3(4431.9-12806.5)$ \\
\hline $\begin{array}{l}\text { Height } \\
(\mathrm{cm})\end{array}$ & $31.7 \pm 2.9(24-35)$ & $\mathrm{RFD}_{\max 2}\left(\mathrm{~N} \cdot \mathrm{s}^{-1}\right)$ & $2998.2 \pm 1124.5(546.8-4938.6)$ \\
\hline PF1 (N) & $740.3 \pm 151.2(479.0-1044.7)$ & $\mathrm{RFD}_{\text {mean } 1}\left(\mathrm{~N} \cdot \mathrm{s}^{-1}\right)$ & $459.7 \pm 114.5(237.6-705.2)$ \\
\hline PF2 (N) & $967.8 \pm 126.2(717.2-1229.5)$ & $\mathrm{RFD}_{\text {mean2 }}\left(\mathrm{N} \cdot \mathrm{s}^{-1}\right)$ & $290.4 \pm 71.9(176.1-500.9)$ \\
\hline $\begin{array}{l}\mathrm{PF} 2 / \mathrm{PF} \\
1\end{array}$ & $1.3 \pm 0.2(1.0-1.8)$ & Force at RFDmax $(\mathrm{N})$ & $136.5 \pm 67.3(18.3-319.3)$ \\
\hline $\begin{array}{l}\text { Tp1 } \\
(\mathrm{ms})\end{array}$ & $168.9 \pm 32.1(115-241)$ & Force at RFD $\max 2(\mathrm{~N})$ & $785.7 \pm 157.5(522.2-1061.5)$ \\
\hline $\begin{array}{l}\mathrm{Tp} 2 \\
(\mathrm{~ms})\end{array}$ & $349.3 \pm 44.3(241-417)$ & $\begin{array}{l}\text { Distance of flexion } \\
(\mathrm{cm})\end{array}$ & $50.6 \pm 5.1(40.8-60.1)$ \\
\hline
\end{tabular}

$V_{\text {max, }}$ maximum velocity in the squat jump; PF1, peak force 1 or the first peak of the forcetime curve; PF2, peak force 2 or the second peak of the force-time curve; Tp1, time from the initiation of force production until the PF1; Tp2, time from the initiation of force production until the PF2; RFD, rate of force development; $\boldsymbol{R}_{\boldsymbol{1}} \boldsymbol{D}_{\max }$, maximum RFD; $\boldsymbol{R}_{\boldsymbol{1}} \boldsymbol{D}_{\max }$, first peak of the RFD-time curve; $R F D_{\max 2}$, second peak of the RFD-time curve; RFD mean1, mean RFD from the initiation of force production until PF1; RFD mean2, mean RFD from the initiation of force production until PF2. 
Table 2

Correlation coefficients $(r)$ between maximum velocity and selected performance variables in the squat jump.

\begin{tabular}{|c|c|c|c|}
\hline PF1 (N) & $0.82^{* * *}$ & $\operatorname{RFD} \max 1\left(\mathrm{~N} \cdot \mathrm{s}^{-1}\right)$ & $0.62^{* * *}$ \\
\hline PF2 (N) & $0.68^{* * *}$ & $\operatorname{RFD} \max _{2}\left(\mathrm{~N} \cdot \mathrm{s}^{-1}\right)$ & $-0.71^{* * *}$ \\
\hline $\begin{array}{c}\text { PF2 } \\
\text { (controlling } \\
\text { for PF1) }\end{array}$ & 0.30 & $\operatorname{RFD}_{\text {mean } 1}\left(\mathrm{~N} \cdot \mathrm{s}^{-1}\right)$ & $0.48^{* *}$ \\
\hline PF2/PF1 & $-0.54^{* *}$ & $\operatorname{RFD}_{\text {mean2 }}\left(\mathrm{N} \cdot \mathrm{s}^{-1}\right)$ & $0.70^{* * *}$ \\
\hline $\mathrm{Tp} 1$ (ms) & 0.20 & Force at RFDmax1 $(\mathrm{N})$ & $0.36^{*}$ \\
\hline Tp2 (ms) & $-0.64^{* * *}$ & Force at RFDmax2 $(\mathrm{N})$ & $0.83^{* * *}$ \\
\hline $\begin{array}{l}\text { Distance of } \\
\text { flexion }(\mathrm{cm})\end{array}$ & -0.33 & $\begin{array}{c}\text { Force at RFDmax2 } \\
\text { (controlling for PF1) }\end{array}$ & $0.40^{*}$ \\
\hline
\end{tabular}

PF1, peak force 1 or first peak of the force-time curve; PF2, peak force 2 or second peak of the force-time curve; Tp1, time from the initiation of force production until the PF1; Tp2, time from the initiation of force production until the PF2; $R F D$, rate of force development; $R_{F} D_{\max }$, maximum RFD; $\boldsymbol{R}_{\boldsymbol{F}} \boldsymbol{D}_{\max 1}$, first peak of the RFD-time curve; $\boldsymbol{R}_{\boldsymbol{B}} \boldsymbol{D}_{\max 2}$, second peak of the RFD-time curve; $\boldsymbol{R} F \boldsymbol{D}_{\text {mean1, }}$ mean RFD from the initiation of force production until PF1; RFD mean2, mean RFD from the initiation of force production until PF2. Significance: ${ }^{*} p \leq 0.05 ;{ }^{* *} p \leq 0.01 ;{ }^{* * *} p \leq 0.001$.

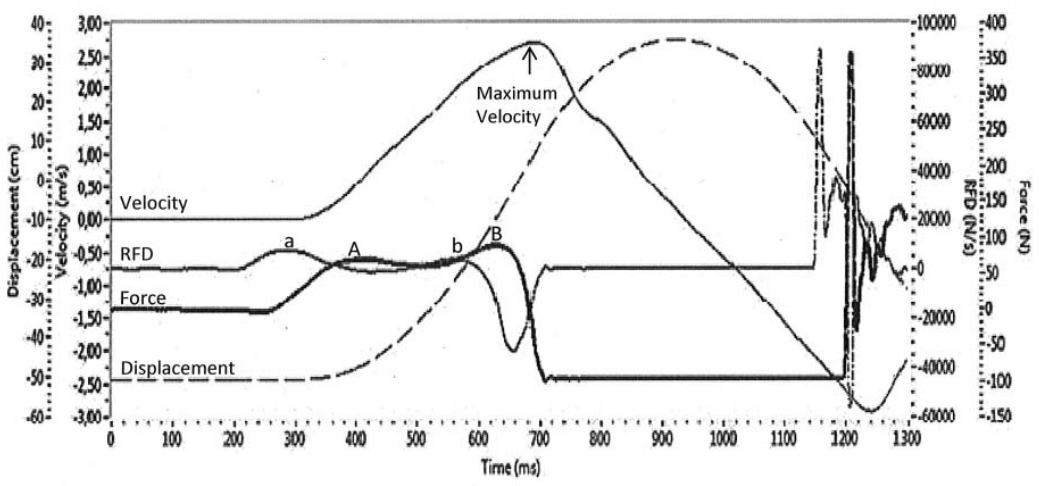

Figure 1

Example of velocity, RFD, force and displacement curves from a representative subject during a squat jump with $17 \mathrm{~kg}$.

RFD = rate of force development; $A=$ peak force $1 ; B=$ peak force 2 ; $a=R F D_{\max 1} ; b=R F D_{\max 2}$. 
Other variable that explains jumping ability is the RFD. Several authors (Kraemer and Newton, 1994; Schmidtbleicher, 1992; Tidow, 1990) claim that the RFD is the determining factor of the activities which require high force production in very little time. In the present study, all analyzed RFD values (RFDmax1, RFDmax2, RFDmean1 and RFDmean2) showed significant correlations with Vmax (Table 2). Although the most relevant analysis of the RFD is the significantly negative relationship between RFDmax 2 and $\operatorname{Vmax}(r=-0.71, \mathrm{p} \leq 0.001)$. These results indicate that the smaller slope of PF2, the higher the Vmax will tend to be. We had not found any study in which an analysis of this variable was made, nor a study where the relationship between the RFD value and performance was negative. To explain the negative sign of this relationship, it should be noted that the RFDmax value which can be achieved in a concentric action is determined by the velocity of displacement: the lower load and therefore higher velocity, the lower the slope which can be reached (Schmidtbleicher, 1992). Therefore, if the same subject has the opportunity to achieve a greater RFD on the slopes of the second peak force, it means that the subject has become slower at that moment, i.e., achieved a lower Vmax (Vmax is reached in less than $100 \mathrm{~ms}$ after the second peak force, Figure 1). Conversely, if the velocity is higher before reaching the second peak force, a lower RFDmax value could be achieved, but Vmax will be higher. Therefore, these relationships between time, force and velocity explain that the lower RFDmax2, the higher Vmax will be. This explains why RFDmax1 is always higher than RFDmax2 (Table 1). The RFDmax1 occurs at the static phase of the movement (jump), in which, naturally, velocity is zero, while the RFDmax2 occurs at a moment near to Vmax.

On the other hand, PF2 is correlated positively with $\operatorname{Vmax}(\mathrm{r}=0.68, \mathrm{p} \leq 0.001)$, which would mean that the higher PF2, the higher Vmax will tend to be. However, in this sense, the present study also provides important information which has not been found in other studies. It is a significantly negative relationship between the ratio $\mathrm{PF} 2 / \mathrm{PF} 1$ and $\operatorname{Vmax}(\mathrm{r}=-0.54, \mathrm{p} \leq 0.01)$. It means that the higher difference between PF2 and PF1, the lower the jump performance will tend to be. Therefore, if the force application starts slowly (applying little force at peak force 1), higher force at peak force 2 could be applied, but the result will tend to be negative, considering that applying more force at peak 2 is possible as the subject reaches that point slower (the lower velocity, the higher force may be applied), and the lower velocity at that moment means a lower Vmax. This result is coherent with a negative relationship between RFDmax 2 and Vmax that we have discussed previously. Therefore, the relationship between PF2/PF1 and Vmax leads us to question the significantly positive relationship between PF2 and Vmax $(r=0.68, p \leq 0.001)$. We should understand that this relationship would be real when the PF1 was also very high, meaning that the difference between PF1 and PF2 was small, but it would not be positive if PF1 was low. Therefore, to test this suggestion, it is necessary to assess the relationship between Vmax and PF2 while controlling for PF1 through a partial correlation. This analysis would allow to determine if the relationship between Vmax and PF2 is independent of PF1. However, as we expected, this did not happen, as when we applied the partial correlation, the relationship between Vmax and PF2 went down clearly, and it went from $r=0.68$ to $r=0.30$ (non-significant). This means that if all PF1 values were equal, there would be no relationship between Vmax and PF2, and then the isolated correlation between these two variables is spurious. From this it follows that a high second peak force is positive if the first peak force is also high. None previous studies had taken into consideration the influence of PF1 to assess the importance of $\mathrm{PF} 2$, and they used the PF2 as a predictor variable of SJ performance, since it is always higher than the PF1, but, as we have just shown, the PF2 does not really present a significant relationship with jump performance.

It is remarkable that the most related variable with Vmax was the force at RFDmax2 $(r=$ $0.83, p \leq 0.001$ ), and even this relationship was not modified practically by controlling for weight, height or distance of knee flexion across a partial correlation, which could indicate that this relationship was independent of any other variable, and therefore, it would be the variable that in major measure explained the jump performance. However, naturally, the applied force to achieve RFDmax2 presents a high 
relationship with the PF2 $(\mathrm{r}=0.89, \mathrm{p} \leq 0.001)$, and therefore, this suggests that probably the relationship between the force at RFDmax2 and Vmax could be determined by PF1, as it was the PF2. Indeed, when we calculated the relationship between the force at RFDmax2 and Vmax while controlling for PF1, this relationship went down from $r=0.83(p \leq 0.001)$ to $r=0.40(p=0.025)$. Therefore, again we observed that the correlation between any possible determinant variables of vertical jump performance could lead to erroneous conclusions if we do not control other variables.

In relation to temporary variables, $\mathrm{Tp} 1$ showed no significant correlation with Vmax. This could be because all subjects used practically the same time to reach the PF1. However, the difference in the performance could be the force that the subject was able to apply. This was corroborated by the correlation between Vmax and RFDmean1 $(r=0.48, p \leq 0.01)$. On the other hand, Tp2 showed a significantly negative correlation with $\operatorname{Vmax}(\mathrm{r}=-0.64, \mathrm{p} \leq 0.001)$, therefore, the higher the Tp2, the slower the Vmax, and therefore, the smaller the vertical jump performance will be. Similar results were shown by González-Badillo and Marques (2010) who also found a significantly negative relationship between time of the concentric phase and CMJ height ( $r=-0.57$ to $-0.87, p<0.05$ ).

In summary, the results of the present study suggest that the best predictor of SJ performance is the applied force at the first peak force. We also observed as a relevant result that the smaller the RFDmax2, the higher the jump performance tends to be. The relationship between the force at RFDmax2 and Vmax is conditioned by the PF1. The smaller the difference between PF1 and PF2, the higher jump performance tends to be.

\section{Practical implications}

A practical application of this study is that the PF1 should be considered as a primary independent variable to explain SJ performance and not the jump maximum peak force (PF2). To determine the predictor variables of the SJ or CMJ, it is necessary to control potential factors that could increase or decrease the real predictive value of each variable. As a future line of research, it would be interesting to assess the evolution of the different variables analyzed and their relationships after a training period.

\section{References}

Ashley CD, Weiss LW. Vertical jump performance and selected physiological characteristics of women. $J$ Strength Cond Res, 1994; 8: 5-11

Bosco C, Luhtanen P, Komi PV. A simple method for measurement of mechanical power in jumping. Eur J App Physiol, 1983; 50: 273-282

Cronin JB, Hansen KT. Strength and power predictors of sports speed. J Strength Cond Res, 2005; 19: 349-357

Dowling JJ, Vamos L. Identification of kinetic and temporal factors related to vertical jump performance. J Appl Biomech, 1993; 9: 95-110

Dowson MN, Nevil ME, Lakomy AM, Nevil AM, Hazeldine RJ. Modeling the relationship between isokinetic muscle strength and sprint running performance. J Sports Sci, 1998; 16: 257-265

González-Badillo JJ, Marques MC. Relationship between kinematic factors and countermovement jump height in trained track and field athletes. J Strength Cond Res, 2010; 24: 3443-3447

Gorostiaga EM, Asiáin X, Izquierdo M, Postigo A, Aguado R, Alonso JM, Ibáñez J. Vertical jump performance and blood ammonia and lactate levels during typical training sessions in elite 400-m runners. J Strength Cond Res, 2010; 24: 1138-1149

Kollias I, Hatzitaki V, Papaiakovou G, Giatsis G. Using principal components analysis to identify individual differences in vertical jump performance. Res Q Exerc Sport, 2001; 72: 63-67

Kraemer WJ, Newton RU. Training for improved vertical jump. Sports Sci Exchange, 1994; 7: 1-12

López-Segovia M, Marques MC, van den Tillaar R, González-Badillo JJ. Relationships between vertical jump 
and full squat power outputs with sprint times in u21 soccer players. J Hum Kinet, 2011; 30: 135-144

Luhtanen P, Komi RV. Segmental contribution to forces in vertical jump. Eur J Appl Physiol Occup Physiol, 1978; 38: 181-188

Mackenzie SJ, Lavers RJ, Wallace BB. A biomechanical comparison of the vertical jump, power clean, and jump squat. J Sports Sci, 2014; 16: 1-10

Marques MC, González-Badillo JJ. Relationship between strength parameters and squat jump performance in trained athletes. Motricidade, 2011; 7: 43-48

Moir G, Shastri P, Connaboy C. Intersession reliability of vertical jump height in women and men. J Strength Cond Res, 2008; 22: 1779-1784

Nagahara R, Naito H, Miyashiro K, Morin JB, Zushi K. Traditional and ankle-specific vertical jumps as strength-power indicators for maximal sprint acceleration. J Sports Med Phys Fitness, 2014; 54: 691-699

Nesser TW, Latin RW, Berg K, Prentice E. Physiological determinants of 40-meter sprint performance in young male athletes. J Strength Cond Res, 1996; 10: 263-67

Reiser RF, Rocheford EC, Armstrong CJ. Building a better understanding of basic mechanical principles through analysis of the vertical jump. Strength Cond J, 2006; 28: 70-80

Sánchez-Medina L, González-Badillo JJ. Velocity loss as an indicator of neuromuscular. Med Sci Sports Exerc, 2011; 43: 1725-1734

Schmidtbleicher D. Training for power events. In: Komi P (ed). Strength and power in sport. London: Blackwell, 381-395; 1992

Tidow G. Aspects of strength training in athletics. New Stud Athlet, 1990; 1: 93-110

Ugarkovic D, Matavulj D, Kukolj M, Jaric S. Standard anthropometric, body composition, and strength variables as predictors of jumping performance in elite junior athletes. J Strength Cond Res, 2002; 16: 227-230

Vandewalle H, Peres G, Monod H. Standard anaerobic exercise tests. Sports Med, 1987; 4: 268-289

Wirth K, Keiner M, Szilvas E, Hartmann H, Sander A. Effects of eccentric strength training on different maximal strength and speed-strength parameters of the lower extremity. J Strength Cond Res, 2015; 29: $1837-1845$

Wisløff U, Castagna C, Helgerud J, Jones R, Hoff J. Strong correlation of maximal squat strength with sprint performance and vertical jump height in elite soccer players. Br J Sports Med, 2004; 38: 285-288

\section{Corresponding author:}

\section{Jorge Ramírez-Lechuga}

Faculty of Sport Sciences, University of Pablo de Olavide, Seville, Spain.

Ctra. de Utrera, km. 141013 Seville, Spain.

Phone: +0034 637828214

E-mail: jrlechuga@upo.es 